\title{
BASIC SIGNAL PROCESSING PRINCIPLES FOR MONITORING OF PERSONS USING UWB SENSORS - AN OVERVIEW
}

\author{
Dušan KOCUR*, Mária ŠVECOVÁ**, Rudolf ZETIK ${ }^{* * *}$ \\ *Department of Electronics and Multimedia Communications, Faculty of Electrical Engineering and Informatics, \\ Technical University of Košice, Letná 9, 04200 Košice, Slovak Republic, e-mail: Dusan.Kocur@tuke.sk \\ **Department of Mathematics and Theoretical Informatics, Faculty of Electrical Engineering and Informatics, \\ Technical University of Košice, Letná 9, 04200 Košice, Slovak Republic, e-mail: Maria.Svecova@tuke.sk \\ *** Fraunhofer Institute for Integrated Circuits IIS, \\ Helmholtzplatz 2, 98693 Ilmenau, Germany, e-mail: rudolf.zetik@iis.fraunhofer.de
}

\begin{abstract}
The paper offers an overview of signal processing approaches that are suitable for monitoring of persons by means of ultrawideband sensors. The "Monitoring of persons" is related to their detection, localization, and tracking and in the case of static persons, also to the monitoring of their vital signs such as the breathing frequency and the heart rate. This paper discusses basic principles in the monitoring of persons who can be moving, static, or changing the character of their movement. In the case of static persons, the paper describes signal processing methods suitable for contactless estimation of their breathing frequency and the heart rate. Monitoring of persons using UWB sensors is attractive in many application scenarios such as emergency, rescue, medical, etc. Therefore, we believe that this comprehensive survey brings a lot of interesting information to researchers and specialists working in the field of contactless monitoring of persons.
\end{abstract}

Keywords: Breathing and heart rate, detection, localization, person monitoring, tracking, UWB sensors/radars, vital signs

\section{INTRODUCTION}

In the last decade, great efforts have been made to develop systems for people monitoring. In this field, special attention has been devoted also to tag-free person localization. The analysis of this issue has shown, that ultrawideband (UWB) sensors (radars) operating in the frequency band DC-5 GHz could be promising tools for tag-free person monitoring [1], [2]. Due to the employment of the ultra-wide bandwidth (usually more than $0.5 \mathrm{GHz}$ ), UWB sensors can provide a fine range resolution, and hence a high accuracy of measurement of the distance between a target and radar antenna array and ultimately, high accuracy of target localization as well [1]. On the other hand, electromagnetic waves occupying such frequency band can penetrate through most non-metallic standard building materials (e.g. concrete, bricks, wood, etc.) with a small enough attenuation. As a result, UWB sensors operating in the frequency band DC-5 GHz allow to detect and to localize people not only for line-of-sight scenarios, but also persons situated behind an non-metallic obstacle (e.g. a wall) or in the environment with a very low optical visibility (e.g. in a snowstorm, dust, smoke, through dense fog and through sparse foliage [3]). Note, that the power of the signal emitted by the mentioned UWB radars is usually below $10 \mathrm{~mW}$. Taking into account the outlined parameters, the key circuitry of the short-range UWB radars can be implemented in a form of application-specific integrated circuits at an acceptable size of radar antennas. Hence, the mentioned radars can be constructed to be lightweight and small-sized, and therefore also flexibly useable in the real operation area.

Due to these properties, short-range UWB sensors can be exploited in many real-life critical situations. Here, the scenarios such as rescue and security operations, critical infrastructures monitoring [1], [2], [4], [5], elderly people monitoring within ambient assisted living programs [6],
[7], baby monitoring focused on the detection of sudden death syndrome, etc., can be listed among them.

As has been shown e.g. in [1], [8], due to the employment of UWB signals, UWB sensors can provide range measurement with a high resolution. This fundamental property of UWB sensors allows for these sensors not only to monitor moving persons (MP) but also to detect some vital signs of static persons (SP). The UWB radars which are mainly focused on a contactless detection of respiration, heart activity, and other biological signals are generally referred to as biometric radars (bio-radars) [9]. Their performance is based on the detection and analysis of backscattered signals caused by movements of the body surface and internal organs. Objects in the human's body, subjected to more or less periodic fluctuations are cardiac muscle and lungs. There are many medical and engineering fields and problems, which could be solved with the help of biometric radars [10]. Here, sleep medicine (somnology) and bedside monitoring [11], [12], functional diagnostics, space medicine [13], in-home monitoring of older adults [7], remote testing of psychological conditions of the persons [14], contactless registration of heartbeat and breathing parameters for burnt patients, newborns, etc., may be considered examples of such areas.

The outlined high application potential of short-range UWB sensors has been the impetus in the last years for relatively extensive research and development in the area of UWB sensor systems designed to people monitor. Analysis of the issue of people monitoring using UWB sensors shows that a key challenge to be addressed in this area is processing of primary radar signals to detect and locate people, and then, if it is requested, to monitor in a contactless way their vital signs (breathing frequency, heart rate). These issues have been studied within the framework of a series of contributions. However, the particular considered methods have been focused usually only on 
partial issues of monitoring of persons (e.g. for MP or SP localization or for breathing frequency and heart rate estimation). In contrast to these approaches, the papers [15]-[20] are creating a unified platform for all mentioned aspects of person monitoring. The platform is based on a set of radar signal processing procedures originally developed for MP and SP localization which are complemented by specific algorithms of estimation of breathing frequency and heart rate. Although a detail description of these methods is beyond this contribution, we would like to introduce a survey of the basic concepts of the methods in this paper. We believe that this short but comprehensive overview of the solutions of particular problems of focused on person monitoring could be attractive for readers interested in this issue.

Following this intention, this paper will have the following structure. In the next section, the basic principles of person detection depending on the style of his/her motion will be summarized. Then, radar signal processing procedures for MP and SP localization and persons moving with an unknown character of motion will be discussed in Sections 3-5. Sections 6-8 will be addressed to the issue of estimation of breathing frequency and heart rate of human beings. And finally, in Conclusion (Section 9), we will point out some challenging problems emerging in the field of person monitoring using UWB sensors.

\section{BASIC PRINCIPLES OF PERSON DETECTION}

In the case of person monitoring by using UWB sensors, raw radar signals (data) gathered by such sensors are represented by a set of the impulse responses of the environment through which the electromagnetic waves emitted by the radar are propagated from transmitting to receiving antenna of radar. This set of impulse responses is usually referred to as radargram [1], [15], [16]. Then, target co-ordinates can be obtained by sequential processing of particular impulse responses or the radargram as a whole. However, due to the power level of signals transmitted by the radar, target-echo-to-noise and clutter ratio (ENCR) is usually very low, and hence simple signal processing methods cannot be used directly to detect and localize persons. Instead, comprehensive radar signal processing procedures have to be applied for that purpose. Note that such radar signal processing procedures consist of a set of radar signal processing phases where each phase is implemented using proper methods of signal processing [15]- [20].

Analysis of the issue of people monitoring using UWB sensors shows that a key challenge to be addressed in this area is the processing of primary radar signals to detect and locate people for the different application scenarios. In the past, two fundamental approaches for human beings localization have been studied.

The former is intent on the localization of so-called MP, i.e. persons moving within the monitored area in such a way, that their coordinates are changing (e.g. a walking person). In this case, the persons are detected based on an observation of the time changes of adjacent impulse responses of the radargram. These changes are identified along the propagation time (fast-time) axis. This concept has led to the development of a UWB radar signal processing procedure for detection, localization, and tracking of MP (MPL) [15].

The latter approach is devoted to the localization of socalled SP, i.e. persons situated, but not moving (e.g. unconscious persons) within the monitored area (i.e. their coordinates are not changing). Breathing of a person is accompanied by his or her respiratory movements, which manifest themselves outwardly in particular by the movement of the chest or by movement of other parts of the body. Note, that respiration is usually the only visible motion activities of SP. Then, the radar signal processing procedure has to be able to detect the respiration motion of a person and hence the person himself/herself. The employment of this idea has led to the design of UWB radar signal processing procedure for detection and localization of SP (SPL) [16].

Many publications dealing with person localization are usually focused either on MP or SP detection and localization. In real scenarios, however, it is usually necessary to locate person changing nature of his/her movement (MP-SP), i.e., a person who has the character of an MP within a certain time interval and, but then, he or she can be considered as an SP in another time interval. An example is a scenario when a person enters an empty room and sits on a chair (i.e. first, he or she is in the role of MP), stays in the room sitting on a chair (then he or she is in the role of SP) and finally leaves the room again as a MP. The characteristic feature of MPL is that it allows detection of MP but is not capable of detecting SP. On the other hand, SPL allows detection of SP but is not capable of detecting MP. Because of that, a radar signal processing procedure based on parallel processing of raw radar data by MPL and SPL supplemented by fusion of data representing the coordinates of targets detected and localized by MPL and SPL can be used for MP-SP detection and localization [17], [18].

Following the above outlined basic principles of MP and SP detection, one can create the following concept of UWB sensor signal processing intended for the person monitoring. This concept is based on the decomposition of possible movement activities of persons to the final set of basic forms of motion, while for detection and localization of the person performing a basic form of motion, a specific procedure for radar signal processing is proposed. And finally, this approach can be complemented by algorithms to be applied for the estimation of breathing frequency and heart rate. Note, these algorithms can be applied for SP only.

The basic classification and definitions of the person types according to the basic forms of their motion is given in Table I [18]. Moreover, motion examples corresponding to the particular types of persons are given in this table too. The set of person types is formally complemented by a state when no person is present in the monitored area (so-called "empty room"). In the next parts of this paper, we will outline basic methods allowing localize the particular types of person and in the case of SP to estimate the basic parameters of their vital signs.

Here we would like to note, that throughout our paper, M-sequence UWB radar employing one transmitting and two receiving antennas is considered as UWB sensor to be applied for the person monitoring. 
Table 1 A classification of the person types according to the character of their motions

\begin{tabular}{|c|c|c|c|}
\hline & Person type & Description of person motion character & Example \\
\hline 1. & $\begin{array}{l}\text { Moving person I } \\
(\mathrm{MP}-\mathrm{I})\end{array}$ & $\begin{array}{l}\text { The person is continuously moving within the } \\
\text { monitored area in such a way that his/her } \\
\text { coordinates are changing. }\end{array}$ & $\begin{array}{l}\text { Walking, running or crawling } \\
\text { person. }\end{array}$ \\
\hline 2. & $\begin{array}{l}\text { Moving person II } \\
\text { (MP-II) }\end{array}$ & $\begin{array}{l}\text { Not motionless person but his/ her coordinates are } \\
\text { not changing. The person is still on the same place, } \\
\text { but his or her limbs (legs, hands, head) or trunk are } \\
\text { in motion. }\end{array}$ & rson waving his hands. \\
\hline 3. & $\begin{array}{l}\text { Static person } \\
\text { (SP) }\end{array}$ & $\begin{array}{l}\text { "Motionless" person situated in the monitored } \\
\text { area. Person's respiration motions are the only } \\
\text { visible form of his/her movement. His/ her } \\
\text { coordinates are not changing. }\end{array}$ & $\begin{array}{l}\text { A sleeping person, unconscious } \\
\text { person. }\end{array}$ \\
\hline 4. & $\begin{array}{l}\text { The person changing of } \\
\text { nature (character) of } \\
\text { his/her movement I } \\
(\mathrm{MP}-\mathrm{SP}-\mathrm{I})\end{array}$ & $\begin{array}{l}\text { The same person may be once regarded as MP and } \\
\text { once again as SP. The person is continuously in } \\
\text { the role of SP over an observation time interval* } \\
\text { with the length } \Delta T_{S P} \leq 30 \mathrm{~s} \text {. }\end{array}$ & $\begin{array}{l}\text { The person walking with } \\
\text { stop/stops. The duration of the } \\
\text { stop (in the next referred to as } T_{S} \text { ) } \\
\text { is maximally } 30 \mathrm{~s} \text { (i.e. } T_{S} \leq 30 \mathrm{~s} \text { ). }\end{array}$ \\
\hline 5. & $\begin{array}{l}\text { The person changing } \\
\text { nature (character) of } \\
\text { his/her movement II } \\
\text { (MP-SP-II) }\end{array}$ & $\begin{array}{l}\text { The same person may be once regarded as MP and } \\
\text { once again as SP. The person is continuously in } \\
\text { the role of SP over an observation time interval* } \\
\text { with the length } \Delta T_{S P}>30 \mathrm{~s} \text {. }\end{array}$ & $\begin{array}{l}\text { The duration of a stop is } \\
\left.\text { minimally } 30 \mathrm{~s} \text { (i.e. } T_{S}>30 \mathrm{~s}\right) \text {. }\end{array}$ \\
\hline 6. & Empty monitored area & There is no person in the monitored area. & Empty room. \\
\hline
\end{tabular}

*Observation time interval: An observation time interval associated with the observation time instant $\tau$ is defined as the interval $\left\langle\tau-\Delta T_{S P}, \tau\right\rangle$. The length of this interval is $\Delta T_{S P}$.

\section{MOVING PERSON LOCALIZATION}

An analysis of MP echo has shown that such signal represents a significantly non-stationary component of the primary radar signals. And hence, a basic principle of MPI and MP-II detection consists in the detection of time changes of adjacent impulse responses of radargram due to non-stationarity of the target echo.

As we have outlined in the previous section, for detection, localization, and tracking of MP-I and MP-II, MPL introduced in [15] can be used. MPL consists of the set of five basic signal processing phases while these phases are implemented using a properly selected signal processing methods. These phases include background subtraction, target detection, time of arrival (TOA) estimation including TOA association, target localization, and target tracking. For their implementation, signal processing methods such as exponential averaging method (background subtraction phase), constant false alarm rate detection (target detection phase), trace connection method (TOA estimation phase), direct computation (target localization phase) and multiple target tracking system (target tracking phase) are recommended. The deeper discussion and description of MPL are behind the scope of the paper. The additional information focused on this topic can be found e.g. in [15], [17] and [18].

\section{STATIC PERSON LOCALIZATION}

A basic principle of SP detection consists usually in the detection of the person's respiratory motions. We note that the term respiratory movement of a human being means a movement of parts of the human body as a result of his or her breathing [16].
Human beings respiration can be considered as a periodical process with a fundamental harmonic frequency $f_{b} \in F_{B}=<0.16 \mathrm{~Hz} ; 1 \mathrm{~Hz}>$, whereby the pick-to-pick value of the chest motion due to the respiration in adults is about $0.4-1.2 \mathrm{~cm} \mathrm{[6],} \mathrm{[12],} \mathrm{[21].} \mathrm{From} \mathrm{the} \mathrm{point} \mathrm{of} \mathrm{view} \mathrm{of}$ localization, these variations can generally be considered negligible. Then, SP can be detected based on the identification of periodical components of the radargram with subtracted background with a fundamental harmonic component from the frequency interval $F_{B}$ for a constant bistatic distance between the target and radar antenna array.

Then, using the outlined principle, SP can be localized using the SPL proposed in [16]. SPL introduced in this contribution consists of the set of five basic signal processing phases such as background subtraction, target echo enhancement, target detection, TOA estimation, and target localization. For the implementation of these phases, exponential averaging method (background subtraction phase), range filtering along the fast-time axis and low-pass filtering along slow-time axis (target echo enhancement phase), trace connection method (TOA estimation phase), and direct computation method (localization phase) can be used.

For a robust detection of persons, it is advantageous to use a two-stage detector consisting of an order statistic constant false alarm rate detector (OS-CFAR) (the first detection stage, its decision statistics are given by the total power of the analysed signal in the frequency band $f_{b} \in F_{B}=<0.16 \mathrm{~Hz} ; 1 \mathrm{~Hz}>$ ) and a comparator with a constant threshold (the second stage of the detector). For the signal power estimation located in $F_{B}$, a Welch periodogram method can be applied [22]. 
Here, we would like to note that an important limiting factor for SP detection is the total time of his/her monitoring. Our experimental experiences supported also by knowledge provided by spectrum analysis theory of periodical signals have shown, that measurement with the length at least 30 s is necessary to detect SP.

Similarly to MPL, a detailed description of SPL is beyond the scope of this contribution and therefore it is not mentioned here. The reader can find it e.g. in [16].

\section{LOCALIZATION OF PERSONS CHANGING NATURE OF HIS / HER MOVEMENT}

In the case of real-life scenarios, the person monitoring system has no a priori information about a type of the person to be monitored. Moreover, persons to be localized are neither perfectly moving nor perfectly static ones. They are usually moving by different styles, which are changing. And hence, we have to deal with the issue of localization of persons which change the nature of their motion.

In the case of localization of persons of unknown type, MPL and SPL can be used concurrently for the processing of the raw radar signals. Then, these procedures allow simultaneously detect and localize of MP and SP. And finally, the coordinates of the targets estimated by these procedures defining the position of the detected persons are subsequently used in the radar display unit to indicate the location of the targets in the monitored area.

A specific situation occurs when a person changes his/her role from SP to MP. For the detection of MP, theoretically, the time interval necessary to measure two impulse responses is sufficient. However, taking into account the transition effects associated with the phases of background subtraction and target detection, it is necessary to measure approximately $10-20$ impulse responses to detect MP, if SP is changing his/her role from SP to MP. It corresponds to a time interval of approximately 1-2 seconds. However, it follows from this that the detection and localization of a person who changes his role from SP to MP may be considered almost instantaneous.

A more complicated situation arises in the case of localization a person who changes his role from MP to SP [18]. For this scenario, two variants should be considered according to Table I. Let us first consider the localization of MP-SP-I. In this case, MP takes the role of SP for during the time interval shorter than $30 \mathrm{~s}$, and therefore, as shown in Section 4, the probability of its detection as SP is low.

To solve this problem, it has been suggested in [18] to use detection of a person's stop based on monitoring of the variance of the impulse responses for the particular observation time instants. In this article, an estimator of the variance of the impulse responses with a subtracted background in a combination with a comparator employing an adaptation of its reference level has been suggested as the mentioned detector. The response of the detector indicating the stop of the person is accepted for the period of cca 30s. In this case, as an estimate of the coordinates of the target, the most recent estimate of the co-ordinate of the person in the MP role is considered. After this time, the person may be detected as SP. In this case, however, it is the detection of MP-SP-II. However, if this does not happen, this status is considered to be the situation where the monitored MP has left the monitored area. A summary of the above-described algorithms provides a radar signal processing procedure for MP-SP-I and MP-SP-II localization (MP-SP-L). A detail description of MP-SP-L can be found in [18].

If no target was detected in the monitored area by the MPL, SPL and MP-SP-L procedures, and at the same time the stop detector does not indicate an MP stop, it can be noticed that there are no targets in the monitored area, which in Table I is formally defined by the NP person type.

\section{CONTACTLESS MONITORING OF BASIC PARAMETERS OF VITAL FUNCTIONS OF PERSONS}

In the field of monitoring of people, in addition to their localization, great attention is nowadays also paid to the non-contact measurement of their respiration rate and heart rate. UWB sensor systems providing this form of human monitoring are often referred to as biometric radars (sensors) or as bio-radars [9]. Comparing the requirements for biometric radar applications and radars applied to the localization of persons has shown that the same sensors can be used to solve the tasks to be met by these radars. Note, however, that specific UWB sensor applications will always lead to specific technical solutions (different antennas, ranges, performance, frequency bands, etc.).

An even greater affinity between the above-mentioned UWB radar groups can be identified in the field of signal processing. This affinity results from the fact that in the case of non-contact measurement of person's vital signs by UWB sensors, a bistatic distance of the monitored person from the radar antenna system must be determined firstly (e.g. [19]). Note that the determination of this distance corresponds to the TOA estimate of the monitored person. Only by determining this distance it is possible to proceed to estimate the frequency of respiratory movements and the heart rate of a person.

The state-of-the-art in the field of biometric radar has shown that biometric radars have been applied only for SP monitoring. For the time being, there have not been yet any published works and results that would indicate the possibility of using UWB sensors to monitor vital signs of MP. Theoretically, in our opinion, such a task could be solvable, but it involves a whole range of unsolved problems. Therefore, we will focus only on the estimation of breathing frequency and heart rate of SP in this paper.

Comparing the range resolution UWB radar (e.g $0.5 \mathrm{~cm}-$ $3 \mathrm{~cm}$ ) and physical dimensions of a human (e.g. $180 \mathrm{~cm} \mathrm{x}$ $60 \mathrm{~cm} \times 80 \mathrm{~cm}$ ) shows that such radar can detect not only one but also several reflections of electromagnetic waves from the human body. That is why we can say that people are complex (distributed) goals in this case.

This moment can be used to monitor the vital signs of the SP by first realizing an estimate of the bistatic distance of the target $\left(R_{0}\right)$. To solve this task, the first to the fourth phase of SPL can be used, i.e., phases such as background subtraction, target echo enhancement, and target detection, with the last phase being the estimate and association of TOA. Then, due to the relatively high range resolution of the UWB radar, it is possible to create the interval of bistatic distances $\Delta R=<R_{0}-s / 2, R_{0}+s / 2>$ in which the monitored person could be located. It is known that the part of the body of a person whose respiratory movements 
are most noticeably observable is the chest. Therefore, the constant $s$ used in the above relationship is generally one of the dimensions of the human chest. Starting from such a defined interval of $\Delta R$, a subset of the radargram can be assembled, including all the radargram components that correspond to the bistatic distances from the $\Delta R$ interval. In the next, this radargram subset will be referred to $K$. In the case of estimating the breathing rate and human heart rate, only the primary radar signals from this set of signals will be processed.

\section{BREATHING FREQUENCY ESTIMATION}

Estimation of the human breathing frequency based on processing of signals measured by the UWB sensor can be accomplished by using signal processing phases such as increasing (enhancing) the power of radargram components due to person respiration motions-to-clutter and noise ratio $\left(E N C R_{B}\right)$, estimating the power spectrum of the signals from the set of signals $K$ obtained as a result of the $E N C R_{B}$ increasing, selection signals with a relatively high level of $E N C R_{B}$, and finally the estimation of respiratory rate itself [19], [20]. In order to increase $E N C R_{B}$, the exponential averaging method (clutter suppression) in combination with the range filtering as well as with the method of filtering the signals from the $K$ set for the case of constant bistatic distances (e.g. by a low-pass filter with a cut off frequency of $1 \mathrm{~Hz}$ ) can be used. The set of signals obtained by that approach will be referred to as $K_{B 1}$.

As mentioned in the previous section, human breathing can be considered as a periodic process, whose fundamental harmonic component has the frequency $f_{b} \in F_{B}=<0.16 \mathrm{~Hz} ; 1 \mathrm{~Hz}>$. Therefore, the next signal processing phase consists in estimating the power spectrum of signals from the $K_{B 1}$ set. To solve this problem, the Welch periodogram method can be used with advantage [22].

At estimating breathing rate, it can be assumed that breathing frequency is the dominant component of the power spectrum of signals from the set of $K_{B 1}$ in the frequency band $F_{B}$. Then, to obtain the best possible estimate of this frequency, it is necessary to choose from the set of signals $K_{B 1}$ its components, which possess a relatively high value of $E N C R_{B}$.

For that purpose, a method based on the evaluation of a ratio of the dominant spectrum component and the average power of the other spectrum components of the signal set of $K_{B 1}$ can be used (peak to average power ratio (PAPR) metrics) [19].

Using this approach, a subset $K_{B 2}$ of the signals from sets $K_{B 1}$ whose components have a relatively high $E N C R_{B}$ value can be obtained. Then, its components are used to get the best possible estimate of the respiration rate of the monitored person from the available set of signals.

And finally, the computation of the power spectrum of signals from the set $K_{B 2}$ using the Welch periodogram is the last step in the respiratory rate estimation process. The dominant frequency of the power spectrum thus obtained, located in the frequency band $F_{B}$, is then used as the estimate of the breathing frequency of the person.

\section{HEART RATE ESTIMATION}

Estimation of human heart rate by the processing of signals measured by the UWB sensor can be made by modifying the method of estimating the human respiration rate described in the previous section. This modification has to reflect finding that the frequency of human heart activity $f_{h}$ is from the interval $F_{H}=<0.8 \mathrm{~Hz} ; 3 \mathrm{~Hz}>$, while the ratio of the power of radargram components due to heart motion-to-clutter and noise power $\left(E N C R_{H}\right)$ is substantially lower than in the case of estimation frequency of human breathing (i.e. as $E N C R_{B}$ ). Note, that the physical background of the detectability of the internal organs of humans (including heart) is in the case of UWB sensor applications based on the dielectric contrast of the human tissues. Some details concerning this issue can be found e.g. in [1], [23], [24].

In addition, the mathematical analysis of the primary radar signal model confirmed also by experimental results, has shown, that the primary radar signal components due to respiratory movements and cardiac activity are periodic but not harmonic signals [21]. As a result, not only their basic harmonic component but also their higher harmonic components can appear in the frequency domain. This finding is particularly important in the case of estimating the heart rate. From the comparison of the frequency intervals $F_{B}$ and $F_{H}$ we can see that the higher harmonic components of the respiratory rate are also found in the frequency band $F_{H}$. An analysis of this problem in [20] has shown that these harmonic components may also have higher power than the frequency component caused by the breathing of the person, and hence the dominant component of the spectrum of signals from the set of $K$ in the frequency band $F_{H}$ may not be the heart rate. Based on this, the method suggested in [20] can be used to estimate the human heart rate based on the processing of signals measured by the UWB radar.

Firstly, using the algorithm described in the previous section, a set of signals $K$ is created. In order to increase the $E N C R_{H}$ of signals contained in this set, it is possible to use the method of exponential averaging (clutter suppression), in combination with the method of filtering impulse responses (e.g., application of a range filter) as well as with filtering of signals from the set $K$ for the case of constant bistatic distances (e.g. by a filter tuned to the frequency band $F_{H}$ ). The set of signals obtained by that approach will be referred to $K_{H}$.

In order to suppress the higher harmonic components of breathing frequency, an adaptive single delay line canceller (SDLC) can be applied to process the signals of the set of $K_{H}$ [21], [25]. Note that for effective implementation of SDCL filtering, it is necessary to know the frequency of human respiration. And finally, filtering using Savitzki and Golay smoothing filters [26] is the last operation applied at this stage. Then, a set of signals $K_{H 1}$ will be the result of this phase of primary radar signal processing (phase of $E N C R_{H}$ enhancement).

The results of this signal processing phase are followed by the phase of estimating the power spectrum of signals from the set $K_{H 1}$ (Welch periodogram), by the phase of the selection of signals with relatively high levels of $E N C R_{H}$ 
(PAPR metric application), and ultimately the heart rate estimation itself $\left(f_{h}\right.$ is estimated as the dominant component of the spectrum of signals from the set of $K_{H 1}$ in the frequency band $F_{H}$ ). Note, the implementation of these phases is similar to those applied in the case of respiratory rate estimation, but the frequency band $F_{B}$ has to be replaced by the frequency band $F_{H}$.

\section{CONCLUSIONS}

In this paper, we have presented a brief macroscopic overview of signal processing methods that are suitable for monitoring of persons by a UWB radar. Under the notion people monitoring, we have included not only localization and tracking of persons but also the estimation of selected vital sign parameters such as their breathing frequency and heart rate. A detailed description of the procedures, methods, and algorithms outlined here is beyond the scope of this contribution. However, the reader advised the papers listed in the references. Many of these papers contain detailed descriptions of their procedures, as well as experimental results.

Despite the great advances in UWB technology and signal processing methods applied for the monitoring of persons, multiple challenging problems are still open. They include e.g. mutual shadowing of targets, identification of false targets, estimation of MP trajectories with large deviations from their true trajectories, insufficient coverage of the monitored area due to radar antenna properties, efficient solution of the impact of the effect of geometric dilution of precision, etc. Solutions of these challenges could result in UWB monitoring systems with much higher robustness than the current systems have. Moreover, reallife applications of UWB sensors may be restricted by valid standards defining especially frequency bands and electromagnetic emission limits for UWB sensor operation (e.g. [27], [28]). The implementation of these standards usually leads to low-levels of ENCR, which decreases the robustness and reliability of UWB sensor performance. The relatively high price of UWB sensors and their antenna systems is another aspect which in our opinion limits their massive deployment.

On the other hand, the state-of-the-art in the field of UWB sensor applications focused on people monitoring has shown that this topic is still very attractive not only for researchers but also for industry and final users. This has been confirmed by the increase in the number of papers and related products of industrial companies. This state can be perceived by the fact that the knowledge from the area of UWB sensor technology acquired at the level of basic research and its subsequent application in applied research begins to create conditions for the prospective mass deployment of UWB sensors in the near future.

\section{ACKNOWLEDGMENTS}

This work was supported by the Slovak Research and Development Agency under the contract No. APVV-150692 and by the Scientific Grant Agency (VEGA) under the contract No. 1/0772/17.

\section{REFERENCES}

[1] SACHS, J.: Handbook of ultra-wideband short-range sensing: theory, sensors, applications, John Wiley \& Sons, 2013.

[2] WithingtON, P. - FLUHLER, H. - NAG, S.: Enhancing Homeland Security with Advanced UWB Sensors, IEEE Microwave Magazine, Vol. 4, No. 3, pp. 51-58, Sept. 2003

[3] YAMAUCHI, B.: All-weather perception for manportable robots using ultra-wideband radar, Proceedings of the 2010 IEEE International Conference on Robotics and Automation (ICRA 2010), Anchorage, Alaska, pp. 3610-3615, May 2010

[4] HUFFMAN, C. - ERICSON, L.: Though-the-Wall Sensors (TTWS) for Law Enforcement: Market Survey, ManTech Advanced Systems International, Inc., 2012.

[5] ReTWis: Look behind the walls, http://www.lokalizacni-systemy.cz/en/retwis-en/.

[6] SACHS, J. - HELBIG, M. - HERRMANN, R. KMEC, M. - SCHILLING, K. - ZAIKOV, E.: Remote Vital Sign Detection for Rescue, Security, and Medical Care by Ultra-Wideband Pseudo-Noise Radar, Ad Hoc Networks, vol. 13, pp. 42-53, 2014.

[7] DIRACO, G. - LEONE, A. - SICILIANO. P.: A Radar-Based Smart Sensor for Unobtrusive Elderly Monitoring in Ambient Assisted Living Applications, Biosensors, Vol. 7, No. 4, 2017.

[8] SACHS, J. - HELBIG, M. - HERRMANN, R. KMEC, M. - SCHILLING, K.: On the Range Precision of UWB Radar Sensors, 11-th International Radar Symposium, pp. 1-4, June 2010.

[9] ZHANG, Y. - CHEN, F. - XUE, H. - LI, Z. - AN, Q. - WANG, J. - ZHANG, Y.: Detection and identification of multiple stationary human targets via bio-radar based on the cross-correlation method, Sensors, Vol. 16, No. 11, pp. 1-12, 2016.

[10] ANISHCHENKO, L. et al.: Bioradiolocation: Methods and Applications, Biomedical Informatics and Technology, Berlin, Heidelberg, Springer, pp. 10 $-28,2014$.

[11] BUGAEV, A. S. et al.: Through wall sensing of human breathing and heart beating by monochromatic radar, Proceedings of the Tenth International Conference on Grounds Penetrating Radar, Vol. 1, pp. 291-294, Jun 2004.

[12] LAZARO, A. - GIRBAU, D. - VILLARINO, R.: Techniques for Clutter Suppression in the Presence of Body Movements during the Detection of Respiratory Activity through UWB Radars, Sensors, Vol. 14, No. 2, pp. 2595-2618, 2014.

[13] BALDI, M. - CERRI, G. - CHIARALUCE, F. EUSEBI, L. - RUSSO, P.: Non-Invasive UWB Sensing of Astronauts' Breathing Activity, Sensors, Vol. 15, No. 1, pp. 565-591, 2015. 
[14] STADERINI, E. M.: An UWB Radar Based Stealthy 'Lie Detector', Ultra-Wideband, Short-Pulse Electromagnetics 6. Boston, MA: Springer US, pp. 537-552, 2003.

[15] KOCUR, D. - ROVŇÁKOVÁ, J. - ŠVECOVÁ, M.: Through Wall Tracking of Moving Targets by MSequence UWB Radar, Towards Intelligent Engineering and Information Technology, I. J. Rudas, J. Fodor, and J. Kacprzyk, Eds. Berlin, Heidelberg: Springer Berlin Heidelberg, pp. 349-364, 2009.

[16] NOVÁK, D. - ŠVECOVÁ, M. - KOCUR, D.: Multiple Person Localization Based on Their Vital Sign Detection Using UWB Sensor, Microwave Systems and Applications, S. Goudos, Ed. InTech, 2017.

[17] KOCUR, D. - NOVÁK, D. - ŠVECOVÁ, M.: UWB Radar Signal Processing for Localization of Persons with the Changing Nature of Their Movement, Sensors \& Transducers Journal, Vol. 207, No. 12, pp. 50-57, Dec 2016.

[18] KOCUR, D. - ŠVECOVÁ, M. - NOVÁK, D.: UWB Radar Based Localization of a Person Changing the Nature of Their Motion State, 2018 International Conference on Radar, pp. 1-6, Aug. 2018.

[19] KOCUR, D. - NOVÁK, D. - DEMČÁK, J.: A joint localization and breathing rate estimation of static persons using UWB radar, 2017 IEEE International Conference on Systems, Man, and Cybernetics (SMC), pp. 1728-1733, Oct. 2017.

[20] KOCUR, D. - ŠVECOVÁ, M. - DEMČÁK, J.: Estimation of Breathing Frequency and Heart Rate by Biometric UWB Radar, 2018 IEEE International Conference on Systems, Man, and Cybernetics (SMC), pp. 1-7, Oct. 2018.

[21] LAZARO, A. - GIRBAU, D. - VILLARINO, R.: Analysis of vital signs monitoring using an IR - UWB radar, Progress In Electromagnetics Research, vol. 100, pp. 265-284, 2010.

[22] PROAKIS, J. - MANOLAKIS, D.: Digital Signal Processing, ser. Prentice Hall international editions. Pearson Prentice Hall, 2007.

[23] TAYLOR, J. D.: Ultrawideband Radar. Application and Design, CRC Press, Boca Raton, 2012.

[24] SAHU, K. N. - NAIDU, D. - SANKAR, K. J.: Characteristics of UWB Propagation through Human Being behind Wall, International Journal of Advanced Research in Electronics and Communication Engineering (IJARECE) Vol. 7, No. 2, pp. 151-157, February 2018.

[25] MAHAFZA, B.: Radar Signal Analysis and Processing Using MATLAB, CRC Press, 2016.
[26] ORFANIDIS, S.: Optimum Signal Processing, Sophocles Orfanidis, 2007.

[27] ETSI TR 103 181-3 V2.1.1 (2019-01). Technical Report. Short Range Devices (SRD) using Ultra Wide Band (UWB); Part 3: Worldwide UWB regulations between 3,1 and 10,6 GHz, ETSI 2019. http://www.etsi.org/standards-search

[28] UWB Regulations. A Summary of Worldwide Telecommunications Regulations governing the use of Ultra-Wideband radio. Version 1.2. Decawave Application Note: APR001, Decawave 2015. https://www.decawave.com/wpcontent/uploads/2018/08/apr001_uwb_worldwide_re gulations_summaryrev1.2.pdf

Received January 24, 2019, accepted April 4, 2019

\section{BIOGRAPHIES}

Dušan Kocur was born in 1961 in Košice, Slovakia. He received his Ing. (M.Sc.) and CSc. (Ph.D.) degrees in Radioelectronics from the Faculty of Electrical Engineering, Technical University of Košice, in 1985 and 1990, respectively. Now, he is the full professor at the Department of Electronics and Multimedia Communications of his Alma Mater. His research interests are radar signal processing, UWB technologies, and their applications.

Mária Švecová was born in 1983 in Svidník, Slovakia. She received her M.Sc. degree in Mathematics from Pavol Jozef Šafárik University in Košice in 2006 and Ph.D. degree in Infoelectronics from the Faculty of Electrical Engineering, Technical University of Košice (TUKE) in 2009. She is an assistant professor at the Dept. of Mathematics and Theoretical Informatics, TUKE. Her research interests include the signal processing for person localization and tracking by UWB sensor network.

Rudolf Zetik was born in 1974 in Košice, Slovakia. He received his Ing. (M.Sc.) and Ph.D. degrees in Radioelectronics from the Faculty of Electrical Engineering, Technical University of Košice, in 1997 and 2001, respectively. In 2002, he joined the Electronic Measurement Research Lab at Ilmenau University of Technology, Germany. There, he habilitated in the area of UWB sensors for surveillance applications in emergency and security situations. Since 2014, he is with Fraunhofer Institute for Integrated Circuits IIS, Germany. He is a team manager of Radar, Sounding and mmWave/THz Systems Group. His research interests include UWB sensors, through-wall radars, real time MIMO channel sounding, localisation and imaging in UWB sensor networks, calibration of mmWave antenna arrays, hybrid (analogdigital) beamforming, localization services in cellular communication networks, etc. 